\title{
Correction to: ADP-ribosylation and NAD+ Utilizing Enzymes
}

\section{Paul Chang}

\section{Correction to:}

Paul Chang (ed.), ADP-ribosylation and NAD+ Utilizing Enzymes:

Methods and Protocols, Methods in Molecular Biology, vol. 1813, https://doi.org/10.1007/978-1-4939-8588-3

The funding information was omitted from the original Chapters 19 and 21. The below text has been added to these chapters respectively.

Chapter 19: This work was supported by a Johns Hopkins Catalyst Award (AKLL) and research grants from the Johns Hopkins University School of Medicine Sherrilyn and Ken Fisher Center for Environmental Infectious Disease (AKLL). The proteomics work was also in part supported by R01GM104135S1 (AKLL and RLM), S10OD021502 (SEO), and T32CA009110 (RLM) from the U.S. National Institutes of Health, and the American Cancer Society Research Scholar Award 129539-RSG-16-062-01-RMC (AKLL).

Chapter 21: This work was supported by a Johns Hopkins Catalyst Award (AKLL) and research grants from the Johns Hopkins University School of Medicine Sherrilyn and Ken Fisher Center for Environmental Infectious Disease (DEG and AKLL). This work is also in part supported by R01GM104135S1 (AKLL and RLM) and T32CA009110 (RLM) from the U.S. National Institutes of Health. 